\title{
Evidence for the adiabatic invariance of the black hole horizon area
}

\author{
Avraham E. Mayo * \\ The Racah Institute of Physics, Hebrew University of Jerusalem, \\ Givat Ram, Jerusalem 91904, Israel
}

(October 5, 2018)

\begin{abstract}
Some examples in support of the conjecture that the horizon area of a near equilibrium black hole is an adiabatic invariant are described. These clarify somewhat the conditions under which the conjecture would be true.
\end{abstract}

\section{INTRODUCTION AND SUMMARY}

Consider the event horizon area of a black hole. Hawking's area theorem [1] indicates that whenever classical fields obeying the weak energy condition are involved, this physical entity must always grow under external disturbances. Be that as it may, it is possible to exhibit an assortment of cases in which the slow application and relaxation of an external disturbance does not necessarily mandate an area increase. This is of course in agreement with the identification of the horizon area and the entropy associated with the black hole [2]. After all, in classical thermodynamics entropy is invariant under slow, reversible changes of a system in thermodynamic equilibrium. This implies a similarity of horizon area to an adiabatic invariant in mechanics. What is an adiabatic invariant?

Consider a Hamiltonian system characterized by a Hamiltonian function $H(p, q ; \lambda)$ depending on a parameter $\lambda$. Suppose that the parameter changes slowly with time. It turns out that in the limit as the rate of change of the parameter approaches 0 , there is a remarkable asymptotic phenomenon: two quantities, generally independent, become functions of each other. For example, consider the motion of a perfectly elastic rigid ball between perfectly elastic walls whose separation $l$ slowly varies. In this case the product $v l$ of the velocity of the ball $v$ and the distance between the walls turns out to be an adiabatic invariant, e.g. if we increase very slowly the separation and then very slowly decrease it to the original value (slow in comparison with the characteristic oscillation), then at the end of this process the velocity of the ball will be the same as it was at the start. Additionally, it turns out that the ratio of the energy $H$ of the ball to the frequency of the oscillation between the walls $\omega$ changes very little under a slow change of the parameter, although the energy and frequency themselves may change a lot. Quantities such as this ratio are called adiabatic invariants. A more precise definition of adiabatic invariant is [3]

Definition. The quantity $I(p, q ; \lambda)$ is an adiabatic invariant of the system

$$
\dot{p}=-\frac{\partial H}{\partial q}, \quad \dot{q}=\frac{\partial H}{\partial p}, \quad H=H(p, q ; \epsilon t) .
$$

if for every $\kappa>0$ there is an $\epsilon_{0}>0$ such that if $\epsilon<\epsilon_{0}$ and $0<t<1 / \epsilon$, then

$$
|I(p(t), q(t) ; \epsilon t)-I(p(0), q(0) ; 0)|<\kappa .
$$

Note that this definition allows for the preservation of the adiabatic invariant with a power-law accuracy (with respect to the small parameter describing the speed of frequency change), in comparison with the exponential accuracy considered to be generic 画.

In quantum terms, one may comprehend the adiabatic invariance of $H / \omega$ by the following arguments. Since it is assumed that during the adiabatic change of the system the perturbations imposed have frequencies very small compared to $\omega$, transitions between states of successive quantum numbers are strongly suppressed. Now, for example, for a harmonic oscillator in a stationary state labeled by quantum number $n, H / \omega=(n+1 / 2) \hbar$. Therefore, the ratio $H / \omega$ is preserved.

A general proof that the horizon area is an "adiabatic invariant" will enable the utilization of Ehrenfest's principle [5]: any classical adiabatic invariant corresponds to a quantum entity with discrete spectrum. Ehrenfest showed that for a quasiperiodic system, all Jacobi action integrals of the form $I=\oint p d q$ are adiabatic invariants. This principle is

*Electronic adsress :Mayo@shum.cc.huji.ac.il 
the backbone of the Bohr-Sommerfeld theory- "old quantum mechanics", where all Jacobi actions, invariant under adiabatic changes, are quantized in integers.

Even so, application of this principle to the problem at hand or to any other problem does not depend on whether the adiabatic invariant can be expressed as a Jacobi action integral or not. In fact, since the Hamilton-Jacobi formalism for black holes is not very well developed, it is not even clear that the horizon area of a black hole can be so expressed. First attempts in this direction were made in the framework of the membrane paradigm [6]. In this framework the problem of slow time dependent perturbations of a black hole is the basis for the membrane description of black hole evolution. This describes the horizon as a viscous conductive two dimensional surface. Recently Parikh and Wilczek [7] have calculated an action for black hole membranes using the Euclidean formulation of Teitelboim [8]. Hence, as matters stand we find it useful to seek insights into the problem using a different approach which is based on the Newman-Penrose formalism as presented in the next section.

Accordingly, application of Ehrenfest's principle will then lead to the spectrum for the horizon area operator. On that account, the object of this work is to present a collection of examples in which the horizon area of the black hole shows signs of being the analogue of a mechanical adiabatic invariant (not necessarily in the form of a Jacobi action integral). Each example is decomposed into a static and dynamical problem. As can be easily understood, in the static case there cannot be any energy flux through the horizon, and thus no area increase. However, the static case serves as a limiting case for the dynamical problem as we take the adiabatic parameter to zero (it is not clear beforehand that this limit actually exists). These examples suggest the existence of a theorem which would state that, classically, under suitably adiabatic changes of a black hole in equilibrium, the area of its event horizon does not change. This would provide a formal motivation for quantizing the black hole in the spirit of the "old quantum theory". This approach has lately received increased attention [9].

The paper is organized as follows. In Sec. II we present the basic theory required to answer the question regarding the circumstances under which the black hole horizon area remains unchanged under the influence of an exterior perturbation. In Sec. [II we discuss the generalization of the result obtained by Bekenstein [10] regarding the influence of scalar charges in the Schwarzschild black hole exterior on the horizon's area to the case in which the black hole is electrically charged. The extreme case of the previous example is discussed in Sec. IV. As a further example we consider in Sec. V the possibility that the charges are distributed inside a de Sitter universe with a Schwarzschild black hole embedded inside it. The influence of the perturbation on the cosmological horizon will be also discussed. In Sec. VI we study in brief the possibility that the hole itself is rotating. Finally, in Sec. VII we examine the influence of an electromagnetic radiation on the horizon area of a Schwarzschild black hole.

\section{THE BASIC IDEA}

Here we shall lay out the basic theory needed to answer the question regarding the circumstances under which the black hole horizon area remains unchanged under the influence of an exterior perturbation.

Consider a small patch of event horizon area $\delta A$; it is formed by null geodesic generators whose tangents vectors are $l^{\alpha}=d x^{\alpha} / d \lambda$, where $\lambda$ is an affine parameter along the generators [11]. The complex conjugate null vectors $m^{\alpha}$ and $\bar{m}^{\alpha}$ together with $l^{\alpha}$ and a fourth real vector $n^{\alpha}$ orthogonal to them which satisfies $l^{\alpha} n_{\alpha}=1$, constitute the Newman-Penrose tetrad which lies at the horizon. $m^{\alpha}$ and $\bar{m}^{\alpha}$ are orthogonal to $l^{\alpha}$ and satisfy $m^{\alpha} \bar{m}_{\alpha}=-1$.

By definition of the convergence of the generators $\rho, \delta A$ changes at a rate

$$
\frac{d}{d \lambda} \delta A=-2 \rho \delta A
$$

Now the convergence, defined by $\rho \equiv l_{\alpha} ; \beta m^{\alpha} \bar{m}^{\beta}$, changes at a rate given by the optical analogue of the Raychaudhuri equation 12 13]

$$
\frac{d \rho}{d \lambda}=\rho^{2}+\sigma \bar{\sigma}+4 \pi T_{\alpha \beta} l^{\alpha} l^{\beta}
$$

where $\sigma \equiv l_{\alpha} ; \beta m^{\alpha} m^{\beta}$ is the shear of the generators and $T_{\alpha \beta}$ is the energy momentum tensor of the matter in the exterior of the black hole. The shear, which measures deformation of the horizon, evolves according to

$$
\frac{d \sigma}{d \lambda}=2 \rho \sigma+C_{\alpha \beta \gamma \delta} l^{\alpha} m^{\beta} l^{\gamma} \bar{m}^{\delta},
$$

where $C_{\alpha \beta \gamma \delta}$ is the Weyl conformal tensor [14]. Utilizing the orthogonality relations of the tetrad base and Einstien's equations one may calculate the Weyl conformal tensor on the horizon 


$$
C_{\alpha \beta \gamma \delta} l^{\alpha} m^{\beta} l^{\gamma} \bar{m}^{\delta}=R_{\alpha \beta \gamma \delta} l^{\alpha} m^{\beta} l^{\gamma} \bar{m}^{\delta}+4 \pi T_{\alpha \beta} l^{\alpha} l^{\beta} .
$$

It will be noticed that to keep the horizon area constant requires $\rho=0$ which by Eqs. (2.2) (2.3) implies that both $C_{\alpha \beta \gamma \delta} l^{\alpha} m^{\beta} l^{\gamma} \bar{m}^{\delta}$ and $T_{\alpha \beta} l^{\alpha} l^{\beta}$ vanish at the horizon. Thus, if initially the convergence and shear were equal to zero, then they would remain so. Vanishing of $C_{\alpha \beta \gamma \delta} l^{\alpha} m^{\beta} l^{\gamma} \bar{m}^{\delta}$ requires that the geometry be quasistationary to prevent gravitational waves, which are quantified by $C_{\alpha \beta \gamma \delta}$, from impinging on the horizon. Thus with a quasistationary geometry, preservation of the horizon area requires

$$
T_{\alpha \beta} l^{\alpha} l^{\beta}=0 \quad \text { on the horizon. }
$$

Note that in the dynamical case one should expect a total area increase which can be calculated by first integrating Eqs. (2.2) (2.3) and then substituting the result in Eq. (2.1). However, since we are mainly interested in the adiabatic character of the horizon area it is sufficient to prove that the rate of change of the horizon area, given by $(d \delta A / d \lambda) / \delta A$ is smaller than the rate of deformation, given by $\sigma$, (note that the comparison is between two quantities with the same dimensions). Now, according to Eq. (2.1) $(d \delta A / d \lambda) / \delta A$ is proportional to $\rho$. Hence, it is sufficient to prove that the differential change in $\rho$ is smaller than the differential change in $\sigma$. Evidently the entire evolution of the various quantities in the problem is governed by the magnitude of the Riemann tensor and the energy-momentum tensor on the horizon. Therefore, according to Eqs. (2.2) (2.3) all that is left to verify in order to prove our claim is that the term $T_{\alpha \beta} l^{\alpha} l^{\beta}$ is of higher order in the adiabatic parameter than the term $R_{\alpha \beta \gamma \delta} l^{\alpha} m^{\beta} l^{\gamma} \bar{m}^{\delta}$.

A quick way to see that it is indeed a true claim goes as follows. The distribution of static perturbators around a static black hole should not cause an area increase (this is but a simple consequence of staticity). This can be realized by noting that the zeroth order term in the adiabatic parameter of $R_{\alpha \beta \gamma \delta} l^{\alpha} m^{\beta} l^{\gamma} \bar{m}^{\delta}$ must vanish. However, in general the first order term would not vanish. For example, such terms appear when a contraction is made between the first order correction to the Riemann tensor and the zeroth order corrections to the tetrad base or vice versa. On the other hand, the contraction of the energy-momentum tensor with the generators of the horizon must be an even function of the adiabatic parameter. This claim is based on the demand that this term should be a semi-positive definite quantity as indicated by the weak energy condition, which is assumed to be obeyed by most of the classical forms of matter found in nature. The lowest order available is of course the second one.

Contrary to the common belief which necessitates an increase of horizon area when changes in the black hole take effect, we shall here examine a variety of processes for which the conditions that keep the horizon area unchanged occur naturally. The rule that seems to emerge is that quasistationary changes of the black hole induced by an external influence will leave the horizon area preserved. This means an "adiabatic theorem" for black holes must exist.

\section{REISSNER-NORDSTRÖM BLACK HOLE DISTURBED BY SCALAR CHARGES}

In this section we extend the result obtained by Bekenstein 10 regarding the influence of scalar charges in the Schwarzschild black hole exterior on the horizon area to the Reissner-Nordström case. As mentioned already in the introduction, this example, as for the following examples, is decomposed into a static and dynamical problem. As can be easily understood, in the static case there cannot be any energy flux through the horizon, and thus no area increase. However the static case serves as a limiting case for the dynamical problem as we take the adiabatic parameter to zero.

\section{A. The Static Problem}

Consider a Reissner-Nordström black hole with the metric

$$
d s^{2}=-\left(1-2 M / r+Q^{2} / r^{2}\right) d t^{2}+\left(1-2 M / r+Q^{2} / r^{2}\right)^{-1} d r^{2}+r^{2}\left(d \theta^{2}+\sin \theta^{2} d \varphi^{2}\right) .
$$

The horizon is located at $r_{\mathcal{H}}=M+\sqrt{M^{2}-Q^{2}}$. If the scalar's sources are weak, one may regard the scalar field, $\Phi$, as a quantity of first order, and conduct a perturbation analysis. The scalar's energy-momentum tensor,

$$
T_{\alpha}^{\beta}=\nabla_{\alpha} \Phi \nabla^{\beta} \Phi-\frac{1}{2} \delta_{\alpha}^{\beta} \nabla_{\gamma} \Phi \nabla^{\gamma} \Phi
$$

will be of second order of smallness. Thus to first order the metric 3.1 is preserved.

To begin with we shall consider first the case in which the scalar charges are static. Then, the field equation outside the scalar's sources can be written in the form 


$$
\frac{\partial}{\partial r}\left(\left(r^{2}-2 M r+Q^{2}\right) \frac{\partial \Phi}{\partial r}\right)-\hat{L}^{2} \Phi=0
$$

where $\hat{L}^{2}$ is the usual squared angular momentum operator. Following an almost identical derivation as in 10 for the Schwarzschild black hole case, one finds the following physical solution

$$
\Phi=\Re \sum_{\ell=0}^{\infty} \sum_{m=-\ell}^{\ell} C_{\ell m} P_{\ell}\left(\frac{r-M}{\sqrt{M^{2}-Q^{2}}}\right) Y_{\ell m}(\theta, \varphi)
$$

where the $Y_{\ell m}$ are the familiar spherical harmonic and $P_{\ell}(x)$ are the well-known Legendre polynomials 15$]$. The (complex) coefficients $C_{\ell m}$ permit us to match the solution to every distribution of sources by the usual methods.

An explanation of the term "physical solution" is in order. For the solution to be physically acceptable, $\Phi$ must not induce any divergences in the invariants of the curvature via Einstein's equations. This means that the invariant $\nabla_{\alpha} \Phi \nabla^{\alpha} \Phi$ must be bounded everywhere (any other invariant is proportional to some power of this one). Hence, if the other independent solutions of Eq. (3.3), furnished by the Legendre associated functions $Q_{\ell}(x)$ (which are singular for $x=0$ ) were kept, this condition would be violated.

Keeping in mind that we are interested in the behavior of the expression $T_{\alpha \beta} l^{\alpha} l^{\beta}$ near the black hole's horizon, we turn now to determine the components of the vector $l^{\alpha}$ in the new geometry. Recall that $l^{\alpha}$ is the tangent to the null generator of the horizon. Furthermore, $l^{\alpha}$ is future directed as well as outgoing. Actually, there is no need for determination of the components of $l^{\alpha}$. Any 3D-hypersurface of the form $\{\forall t, r=$ const $\}$ has a tangent $\tau^{\alpha}=\delta_{t}{ }^{\alpha}$ with norm $-\left(1-2 M / r+Q^{2} / r^{2}\right)$ as well as the normal $\eta_{\alpha}=\partial_{\alpha}(r-$ const $)=\delta_{\alpha}{ }^{r}$ with norm $\left(1-2 M / r+Q^{2} / r^{2}\right)$. The linear combination of these two vectors, $N^{\alpha} \equiv \tau^{\alpha}+\left(1-2 M / r+Q^{2} / r^{2}\right) \eta^{\alpha}$ is obviously null, and as $r \rightarrow r_{\mathcal{H}}$, both its covariant and contravariant forms remain well defined. Hence, it must be proportional to $l^{\alpha}$. Note that just like $l^{\alpha}, N^{\alpha}$ is future directed as well as outgoing. Applying the new found $N^{\alpha}$ and substituting the energy-momentum tensor for the scalar field, Eq. (3.2) one is able to write

$$
\lim _{r \rightarrow r_{\mathcal{H}}} T_{\alpha \beta} N^{\alpha} N^{\beta}=\lim _{r \rightarrow r_{\mathcal{H}}}\left(N^{\alpha} \nabla_{\alpha} \Phi\right)^{2}=\lim _{r \rightarrow r_{\mathcal{H}}}\left(\left(1-2 M / r+Q^{2} / r^{2}\right) \partial \Phi / \partial r\right)^{2}=0 .
$$

Equation (3.5) implies that the horizon area is left unaffected by the presence of the static perturbations in the outer region of a Reissner-Nordström black hole. This is essential if the area is an adiabatic invariant under dynamical perturbations of the black hole as is shown in the next subsection.

\section{B. The Time Dependent Problem}

The time dependent scalar equation in the background Reissner-Nordström spacetime is

$$
-\frac{r^{4}}{\left(r^{2}-2 M r+Q^{2}\right)} \frac{\partial^{2} \Phi}{\partial t^{2}}+\frac{\partial}{\partial r}\left(\left(r^{2}-2 M r+Q^{2}\right) \frac{\partial \Phi}{\partial r}\right)-\hat{L}^{2} \Phi=0 .
$$

In analogy with Eq. (3.4) we now look for a solution of the form

$$
\Phi=\Re \int_{0}^{\infty} d \omega \sum_{\ell=0}^{\infty} \sum_{m=-\ell}^{\ell} C_{\ell m}(\omega) f_{\ell}(\omega, r) Y_{\ell m}(\theta, \varphi) e^{-\imath \omega t} .
$$

In terms of Wheeler's generalized "tortoise" coordinate [14], defined by $d r^{*} / d r=\left(1-2 M / r+Q^{2} / r^{2}\right)^{-1}$ for which the horizon resides at $r^{*}=-\infty$, the equation satisfied by the new radial function $H_{\ell \omega}\left(r^{*}\right) \equiv r f_{\ell}(\omega, r)$ is a Schrödinger type equation

$$
\begin{aligned}
& \left(-\frac{d^{2}}{d r^{* 2}}+V_{\ell}\left(r^{*}\right)\right) H_{\ell \omega}=\omega^{2} H_{\ell \omega} \\
& V_{\ell}\left(r^{*}\right)=\left(1-\frac{2 M}{r}+\frac{Q^{2}}{r^{2}}\right)\left(\frac{2 M}{r^{3}}-\frac{2 Q^{2}}{r^{4}}+\frac{\ell(\ell+1)}{r^{2}}\right) .
\end{aligned}
$$

Here $V_{\ell}\left(r^{*}\right)$, with $r$ considered a function of $r^{*}$, is a curvature induced potential barrier [16]. The exact position of the peak depends on $M, Q$ and $\ell$ in general. When $Q=0$, the peak occurs between $r=3 M / 8(\ell=0)$, and $r=3 M$ 
$(\ell \rightarrow \infty)$; when $|Q|=M$ the maximum is at $r=2 M$ for all $\ell$. The height of the potential is inversely proportional to $M^{2}$ : for $Q=0$, the maximum is $V_{0, \max }=27 /\left(1024 M^{2}\right), V_{\ell, \max }=\ell(\ell+1) /\left(27 M^{2}\right)$ as $\ell \rightarrow \infty$; for $|Q|=M$, $V_{\ell, \max }=(1+2 \ell(\ell+1)) /\left(32 M^{2}\right)$. Note that as we approach the horizon the potential decays exponentially with a vanishing value on the horizon.

The analogy between Eq. (3.8) and the Schrödinger eigenvalue equation calls for the following analysis of the effects of distant scalar sources on the black hole horizon [14]. Waves with "energy" $\omega^{2}$ on their way in from a distant source run into a positive potential barrier. Therefore, waves with any $\ell$ and $\omega^{2}$ smaller then the maximal hight of the barrier, coming from sources at $r \gg 3 M$, have to tunnel through the potential barrier to get near the horizon. In due course, the wave amplitudes that penetrate to the horizon are small fractions of the initial amplitudes, most of the waves being reflected back. This means that adiabatic perturbations by distant sources perturb the horizon very weakly. Thus one would not expects significant growth of the horizon area from scalar perturbations originating in distant sources.

What if the scalar's sources are moved into the region $r_{\mathcal{H}}<r<3 M$ inside the barrier? They will now be able to perturb the horizon; do they change its area? To answer these questions, we look for the solutions of Eq. (3.8) in the region near the horizon where the potential is small compared to $\omega^{2}$.

But before undertaking this task one should note that as $\omega$ tends to zero the sources must also be moved closer and closer to the horizon since the inside of the potential barrier moves in towards the horizon. This suggests that there are in fact two time scales in the problem; one due to the time scale of the sources and a second one due to the rate of change of their position. However, since the potential decays exponentially as we come closer and closer to the horizon this means that we can lower $\omega$ without changing the location of the sources very much.

Consequently we turn to find the solutions to Eq. (3.8). According to the theory of linear second order differential equations they are of the form

$$
H_{\ell \omega}\left(r^{*}\right)=\exp \left( \pm \imath \omega r^{*}\right) \times\left[1+O\left(1-2 M / r+Q^{2} / r^{2}\right)\right] .
$$

The Matzner boundary condition 17] that the physical solution be an ingoing wave as appropriate to the absorbing character of the horizon selects the sign in the exponent as negative. Hence the typical term in $\Phi$ is

$$
\frac{1+O\left(1-2 M / r+Q^{2} / r^{2}\right)}{r} P_{\ell}(\cos \theta) \cos \psi ; \quad \psi \equiv \omega\left(r^{*}+t\right)-m \varphi
$$

As can be easily verified, invariants of the curvature associated with this solution [all proportional to $\left(\nabla_{\alpha} \Phi \nabla^{\alpha} \Phi\right)^{k}$ ] are bounded. For example, consider a $\Phi$ composed of a single mode as in Eq. (3.10). An explicit calculation on the Reissner-Nordström background using $d r^{*} / d r=\left(1-2 M / r+Q^{2} / r^{2}\right)^{-1}$ gives

$$
\nabla_{\alpha} \Phi \nabla^{\alpha} \Phi \propto \frac{m^{2} P_{\ell}^{2} \sin ^{2} \psi}{r^{4} \sin ^{2} \theta}+\left(\frac{d P_{\ell}}{d \theta}\right)^{2} \frac{\cos ^{2} \psi}{r^{4}}+\frac{\omega \sin (2 \psi)}{r^{3}} P_{\ell}^{2}+\cdots,
$$

where "..." here and henceforth denote terms that vanish as $r$ goes to $r_{\mathcal{H}}$. This expression is obviously bounded at the horizon. By induction it is possible to show that a $\Phi$ which is the sum of several modes is bounded on the horizon.

Finally we turn our attention to the question whether the horizon area is left unchanged by the perturbation. As with the static case, the expression which governs the extent by which the horizon area increases is proportional to

$$
T_{\alpha \beta} N^{\alpha} N^{\beta}=\left(\partial \Phi / \partial t+\left(1-2 M / r+Q^{2} / r^{2}\right) \partial \Phi / \partial r\right)^{2}
$$

If one now substitutes a $\Phi$ made up of a single mode like in Eq. (3.10), one finds out that

$$
T_{\alpha \beta} l^{\alpha} l^{\beta} \propto \frac{\omega^{2} P_{\ell}^{2} \sin ^{2} \psi}{r^{2}}+\cdots .
$$

In conclusion, on the one hand the amount by which the shape of the horizon is perturbed when the scalar field sources are moved inside the potential barrier does not vanish as the perturbation changes slowly. This quantity is measured by the complex shear as defined in the previous section. According to Eqs. (2.2) (2.3), the evolution of the shear is governed by the value of the Weyl tensor on the horizon. This can be expressed by the Riemann tensor and the energy momentum tensor on the horizon [see Eq. (2.4)]. Now, the metric perturbation, via Einstein's equations, in general must be linear in the magnitude of the invariant $\nabla_{\alpha} \Phi \nabla^{\alpha} \Phi$ (after all Einstein's equations have $T_{\alpha \beta}$ as source, not $\left.T_{\alpha}^{\gamma} T_{\gamma}{ }^{\beta}\right)$. By Eq. (3.11) this perturbation is of order $O\left(\omega^{0}\right)$ generically, and of $O(\omega)$ in the monopole case. Clearly, the zeroth order of perturbation $O\left(\omega^{0}\right)$, corresponds to the static case which was already dealt with previously. Therefore, the dynamic deformation (to be compared with static deformation) is of order $O(\omega)$. On the other hand we have shown that the rate of change of the horizon area is of order $O\left(\omega^{2}\right)$, so that as $\omega \rightarrow 0$ the change in area becomes entirely negligible on the scale of the perturbation. 


\section{EXTREME REISSNER-NORDSTRÖM BLACK HOLE DISTURBED BY SCALAR CHARGES}

It is a well-known fact that the area of an extreme Reissner-Nordström black hole is not invariant under the absorption of a point particle [9, 10]. But as we shall prove now the horizon area of an extreme Reissner-Nordström black hole is indeed an adiabatic invariant under scalar field-black hole interaction.

\section{A. The Static Problem}

The scalar field equation in the extreme Reissner-Nordström background for a time independent situation is

$$
\frac{\partial}{\partial r}\left((r-M)^{2} \frac{\partial \Phi}{\partial r}\right)-\hat{L}^{2} \Phi=0
$$

with the general solution

$$
\Phi=\Re \sum_{\ell=0}^{\infty} \sum_{m=-\ell}^{\ell}\left(\frac{A_{\ell m}}{(r-M)^{\ell+1}}+B_{\ell m}(r-M)^{\ell}\right) Y_{\ell m}(\theta, \varphi) .
$$

The complex coefficients $A_{\ell m}$ and $B_{\ell m}$ enable us to match the solution to any distributions of sources by the usual methods. Now, since we are in search of a solution which is regular on the horizon [recall that invariants such as $\left(\nabla_{\alpha} \Phi \nabla^{\alpha} \Phi\right)^{k}$ must be well behaved there], we must make all the coefficients $A_{\ell m}$ vanish. Thus in the inner region of the black hole exterior (inwards from the sources) we have, to first order in perturbations theory, the exact solution

$$
\Phi=\Re \sum_{\ell=0}^{\infty} \sum_{m=-\ell}^{\ell} B_{\ell m}(r-M)^{\ell} Y_{\ell m}(\theta, \varphi) .
$$

As with the non-extreme case we shall employ the vector $N^{\alpha}$. In the new geometry, $N^{\alpha}=\tau^{\alpha}+(1-M / r)^{2} \eta^{\alpha}$. The change in the horizon area as measured by

$$
\lim _{r \rightarrow M} T_{\alpha \beta} N^{\alpha} N^{\beta}=\lim _{r \rightarrow M}\left(N^{\alpha} \nabla_{\alpha} \Phi\right)^{2}=\lim _{r \rightarrow M}\left((1-M / r)^{2} \partial \Phi / \partial r\right)^{2}
$$

obviously vanishes. Hence, the horizon area is left unaffected by the perturbation.

\section{B. The Time Dependent Problem}

When the sources are moved slowly from infinity into the immediate surroundings of the black hole horizon, we must retain the time derivatives in the scalar field equation

$$
-\frac{r^{4}}{(r-M)^{2}} \frac{\partial^{2} \Phi}{\partial t^{2}}+\frac{\partial}{\partial r}\left((r-M)^{2} \frac{\partial \Phi}{\partial r}\right)-\hat{L}^{2} \Phi=0 .
$$

In analogy with the derivations in the previous sections, one should look for solution of the form

$$
\Phi=\Re \int_{0}^{\infty} d \omega \sum_{\ell=0}^{\infty} \sum_{m=-\ell}^{\ell} C_{\ell m}(\omega) f_{\ell}(\omega, r) Y_{\ell m}(\theta, \varphi) e^{-\imath \omega t} .
$$

As usual, it is possible to transform the last equation with the aid of the transformations $r^{*}=r+2 M \ln (r / M-1)-$ $M /(r / M-1)$ and $H_{\ell \omega}\left(r^{*}\right)=r f_{\ell}(\omega, r)$ into

$$
\begin{aligned}
& \left(-\frac{d^{2}}{d r^{* 2}}+V_{\ell}\left(r^{*}\right)\right) H_{\ell \omega}=\omega^{2} H_{\ell \omega}, \\
& V_{\ell}\left(r^{*}\right) \equiv\left(1-\frac{M}{r}\right)^{2}\left(\frac{2 M}{r^{3}}-\frac{2 M^{2}}{r^{4}}+\frac{\ell(\ell+1)}{r^{2}}\right) .
\end{aligned}
$$


As in the non-extremal case, when the sources are moved through the potential barrier into the region where $\omega^{2}$ is much greater than the potential, one can look for a solution of the form

$$
H_{\ell \omega}\left(r^{*}\right)=\exp \left( \pm \imath \omega r^{*}\right) \times\left[1+O(1-M / r)^{2}\right] .
$$

Again, the Matzner boundary conditions select the sign in the exponent as negative. Hence, a typical term in $\Phi$ is as in Eq. (3.10) (with M equal to Q). As with the non-extreme case it can be easily verified that the invariant $\nabla_{\alpha} \Phi \nabla^{\alpha} \Phi$ is bounded on the horizon, and is generally of order $O\left(\omega^{0}\right)$. Finally the term $T_{\alpha \beta} N^{\alpha} N^{\beta}$ reads

$$
T_{\alpha \beta} N^{\alpha} N^{\beta}=\left(\partial \Phi / \partial t+(1-M / r)^{2} \partial \Phi / \partial r\right)^{2}
$$

and, after substitution of $\Phi$ as in Eq. (3.10) (with M equal to Q), it is plain to see that our former conclusion concerning the non-extreme case is recovered.

\section{Disturbing A STATIC BLACK HOLE IN A DE SITTER UNIVERSE}

As a last example of processes in which the horizon area of a static black hole is left unchanged by an external scalar disturbance, we now consider the case in which scalar charges are distributed in a de Sitter universe with a Schwarzschild black hole embedded inside it. This spacetime can be described by the metric

$$
d s^{2}=-\left(1-2 M / r-\frac{\Lambda}{3} r^{2}\right) d t^{2}+\left(1-2 M / r-\frac{\Lambda}{3} r^{2}\right)^{-1} d r^{2}+r^{2}\left(d \theta^{2}+\sin \theta^{2} d \varphi^{2}\right)
$$

where $M$ is the mass of the black hole, and $\Lambda$ is the cosmological constant. This spacetime is characterized by two horizons which correspond to the two positive roots of the cubic equation, $g_{t t}(r)=0$. If $9 M^{2} \Lambda<1$ there are three real roots. One can be attributed to the black hole horizon, $r_{B H}=r_{B H}(M, \Lambda)$, while the other one is the cosmological horizon, $r_{C}=r_{C}(M, \Lambda)$. The third root $r_{0}$ is always negative and thus unphysical. If $9 M^{2} \Lambda=1$, the two horizons coalesce and are located at $r=(3 M / \Lambda)^{1 / 3}$. In the case where $9 M^{2} \Lambda>1$, we have the unphysical situation in which the mass of the hole exceeds the energy associated with the cosmological constant.

The shape of the effective potential that a scalar converging wave experience when it is propagating in this spacetime is

$$
V_{\ell}\left(r^{*}\right)=\left(1-\frac{2 M}{r}-\frac{\Lambda}{3} r^{2}\right)\left(\frac{2 M}{r^{3}}-\frac{2}{3} \Lambda+\frac{\ell(\ell+1)}{r^{2}}\right) .
$$

In order to test our hypothesis regarding the adiabatic invariance of the black hole horizon area, we must consider the propagation of waves in the region between the black hole horizon and the location of the maximum of the potential barrier. This is due to an assumption that the squared frequency of the waves is small compared to the height of the potential barrier. Thus, waves which propagate outside this region will have no effect on the black hole's horizon. This problem was dealt with in [10] and a generalization to the charged case was presented in the previous sections. Therefore, we shall concern ourselves only with the effect of scalar waves on the cosmological horizon $r_{C}$, namely the effect of the waves in the region between the potential barrier and the cosmological horizon.

For brevity we consider only the time dependent problem. As usual, we can solve the scalar field equation by a separation of variables. Thus, after expanding the time dependence in Fourier modes and the angular dependence in spherical harmonics, the radial part of the wave equation can be written in term of the difference of the roots of the cubic equation $g_{t t}(r)=0 ; \gamma \equiv r_{C}-r_{0}, \delta \equiv r_{C}-r_{B H}$, and the radial location of the cosmological horizon compared to the radial distance to the cosmological horizon, namely $\epsilon \equiv r_{C}-r$,

$$
\frac{d}{d \epsilon}\left(\left(r_{C}-\epsilon\right) \Delta \frac{d R}{d \epsilon}\right)+\left(\ell(\ell+1)+\frac{\left(r_{C}-\epsilon\right)^{3} \omega^{2}}{\Delta}\right) R=0
$$

where $\Delta \equiv \Lambda(\gamma-\epsilon)(\delta-\epsilon) \epsilon / 3$. Note that $\gamma, \delta$ and $\epsilon$ are all positive semi definite by definition.

In light of the previous derivation it is obvious that the problem has two competing scales; the proximity to the cosmological horizon, denoted here by $\epsilon$, and the distance between the two horizons, denoted here by $\delta$. In the beginning we will assume that the black hole horizon is far from the cosmological horizon and that the sources are placed in a close distance from the cosmological horizon, namely $r_{B H} \ll r \leq r_{C}$. Later we will consider the possibility that the two horizons are on the verge of coalescence, $\delta \rightarrow 0$.

Therefore, Eq. (5.3) can be approximated: 


$$
\epsilon^{2} \frac{d^{2} R}{d \epsilon^{2}}+\epsilon \frac{d R}{d \epsilon}+\left(\frac{3 r_{C} \omega}{\Lambda \gamma \delta}\right)^{2} R \simeq 0
$$

This is an Eüler equation with the two independent solutions $R(\epsilon)=N \exp ( \pm \imath \eta \ln \epsilon)$, where $N$ is an arbitrary constant and $\eta=3 r_{C} \omega / \Lambda \gamma \delta$. Now, since the cosmological horizon is situated at $\epsilon=0^{+}$, the solution with the plus sign should be discarded while the solution with the minus sign should be retained in accordance with the fact that only ingoing waves exist at the one-way membrane $r=r_{C}$. Therefore, $\Phi$ consists of terms such as

$$
P_{\ell}(\cos \theta) \cos \psi, \quad \psi \equiv \omega(\eta \ln \epsilon+t)-m \varphi .
$$

It can be easily verified that the above solution does not cause any divergences in the invariants of the curvature. Hence we can turn now to the problem of verifying that the increase in the area of the cosmological horizon is at the same rate as the one found in the previous examples, namely proportional to $\omega^{2}$, while keeping in mind that the perturbation to the metric is proportional only to $\omega$ (see Sec. III). To do so, we just have to calculate expressions such as the ones in Eq. (3.12) with $N^{\alpha}$ appropriate to the new spacetime.

$$
T_{\alpha \beta} N^{\alpha} N^{\beta}=\left(\partial \Phi / \partial t+\left(1-2 M / r-\frac{\Lambda}{3} r^{2}\right) \partial \Phi / \partial r\right)^{2} .
$$

If one now substitutes a $\Phi$ made up of a single mode like in Eq. (5.5), one concludes that

$$
T_{\alpha \beta} l^{\alpha} l^{\beta} \propto \omega^{2} P_{\ell}^{2} \sin ^{2} \psi+\cdots
$$

This result agrees with the ones obtained in the previous situations.

Consider now a series of cases in which the separation between the black hole horizon and the cosmological horizon $\delta$ is gradually diminished. As long as $\delta>0$, the potential barrier maintains its shape, and a weak scalar wave impinging on the black hole horizon can have no effect on the cosmological horizon and vice versa. If this is so, then our conclusion still holds: the black hole and cosmological horizons are left unchanged by the perturbation. In the limit where $\delta \rightarrow 0$ the potential barrier, which separates the two horizons, disappears and the wave can either propagate between the innermost side of the horizon and the singularity, or in the region beyond the cosmological horizon. Both cases will be left for a future discussion. It is important to understand that $\delta$ is not a dynamical parameter in the sense that for a given spacetime $\delta$ is determined uniquely. However, it has a significant influence on the magnitude of the sources that may be used in the adiabatic process. This concludes the example.

\section{KERR BLACK HOLE DISTURBED BY AN AXIALLY SYMMETRIC ARRAY OF SCALAR CHARGES}

In the previous examples we considered a static black hole disturbed by quasistatic perturbation. Now we shall study in brief the possibility that the hole itself is rotating. To simplify the calculation, we assume that the scalar charges are arranged in a configuration with cylindrical symmetry. We work in Boyer-Lindquist coordinates with the metric

$$
\begin{aligned}
d s^{2}= & -\left(1-\frac{2 M r}{\rho^{2}}\right) d t^{2}-4 \frac{M a r \sin \theta^{2}}{\rho^{2}} d \phi d t \\
& +\sin \theta^{2}\left(r^{2}+a^{2}+\frac{2 M r a^{2}}{\rho^{2}} \sin \theta^{2}\right) d \varphi^{2}+\frac{\rho^{2}}{\Delta} d r^{2}+\rho^{2} d \theta^{2}
\end{aligned}
$$

where $\Delta=r^{2}-2 M r+a^{2}$ and $\rho^{2}=r^{2}+a^{2} \cos \theta^{2} . M$ is the mass of the hole and $a$ is its specific angular momentum.

As with the static spacetime case, we begin by writing down the scalar field equation in Kerr spacetime. The details of the solution of the scalar wave equation in a Kerr background without any assumptions on the scalar field can be found in [18]. Assuming that the scalar field is time independent and has axial symmetry, one obtains an equation which is remarkably similar to the equation derived for the scalar field in the static spherical spacetime. The arguments we used in the static spherical spacetime are still applicable here. Therefore, we just write the physical solution

$$
\Phi=\Re \sum_{\ell=0}^{\infty} C_{\ell} P_{\ell}\left(\frac{r-M}{\sqrt{M^{2}-a^{2}}}\right) P_{\ell}(\cos \theta) .
$$


As before, it is possible to use the complex coefficient $C_{\ell}$ in the matching of the solution to any charge configuration with said symmetry.

Now, in order to draw a conclusion regarding the adiabatic invariance of the horizon area, we look at the term $T_{\alpha}^{\beta} N^{\alpha} N_{\beta}$ where $N^{\alpha}$ is proportional to the tangent of the null generator of the horizon which in Boyer-Lindquist coordinates reads 14

$$
\begin{aligned}
& N^{t}=1, \\
& N^{r}=\frac{\Delta}{r^{2}+a^{2}}, \\
& N^{\theta}=0, \\
& N^{\varphi}=\frac{a}{r^{2}+a^{2}} .
\end{aligned}
$$

Since $N^{\alpha}$ is null, namely $N^{r} N_{r}=-\left(N^{t} N_{t}+N^{\phi} N_{\phi}\right)$, we can write

$$
T_{\beta}^{\alpha} N^{\beta} N_{\alpha}=\left(T_{t}^{t}-T_{r}^{r}\right) N^{t} N_{t}+\left(T_{\varphi}^{\varphi}-T_{r}^{r}\right) N^{\varphi} N_{\varphi}
$$

However, because of the symmetries, the terms $T_{t}^{t}-T_{r}^{r}$ and $T_{\phi}^{\phi}-T_{r}^{r}$ are both equal to $-\nabla_{r} \Phi \nabla^{r} \Phi$. Thus, $T_{\beta}^{\alpha} N^{\beta} N_{\alpha}$ can be reduced to $\left(N^{r} \partial \Phi / \partial r\right)^{2}=\left(\partial \Phi / \partial r \Delta /\left(r^{2}+a^{2}\right)\right)^{2}$. Since $\Phi$ is analytic on the horizon, defined by $\Delta\left(r_{\mathcal{H}}\right)=0$, the horizon area is left unchanged by the perturbation.

The story becomes more interesting when one considers the time dependence of the sources. As usual, we make a separation ansatz which is given by the eigenfunctions appropriate for an axially symmetric and stationary background geometry, namely, $\Phi_{\ell \omega}=R_{\ell \omega}(r) \Theta_{\ell}(\theta) e^{ \pm \imath \omega}$. By the usual argument we find the separated homogeneous equations, with separation constant $\lambda_{\ell}[18]$,

$$
\begin{aligned}
\Delta \frac{d}{d r}\left(\Delta \frac{d R_{\ell}}{d r}\right)+\left(r^{2}+a^{2}\right)^{2} \omega^{2} R_{\ell} & =\left(\lambda_{\ell}+\omega^{2} a^{2} \Delta\right) R_{\ell} \\
\frac{1}{\sin \theta} \frac{d}{d \theta}\left(\sin \theta \frac{d \Theta_{\ell}}{d \theta}\right)+a^{2} \omega^{2} \cos \theta^{2} \Theta_{\ell} & =-\lambda_{\ell} \Theta_{\ell} .
\end{aligned}
$$

The second equation is the same as the flat-space angular spheroidal equation with $m=0$. Its eigenvalues are denoted by $\lambda_{\ell}$ and its eigenfunctions $S_{\ell}(-\imath a \omega, \cos \theta)$. These eigenfunctions form a discrete set and go over into Legendre polynomials in the limit $a \omega \rightarrow 0$. The integer $\ell$ has its standards range, but the eigenvalues $\lambda_{\ell}$ cannot be analytically expressed in terms of $\ell[15]$.

The radial equation can be written as a one-dimensional equation with an effective potential by defining a new radial function and a new radial coordinate,

$$
H_{\ell \omega}\left(r^{*}\right)=\sqrt{r^{2}+a^{2}} R_{\ell}(\omega, r), \quad d r^{*}=\frac{r^{2}+a^{2}}{\Delta} d r .
$$

We then find

$$
\begin{aligned}
& \left(-\frac{d^{2}}{d r^{* 2}}+V_{\ell, \omega}\left(r^{*}\right)\right) H_{\ell \omega}=\omega^{2} H_{\ell \omega} \\
& V_{\ell, \omega}\left(r^{*}\right) \equiv \frac{\Delta}{\left(r^{2}+a^{2}\right)^{2}}\left(\left(\lambda_{\ell}+\omega^{2} a^{2}\right)+\frac{3 r^{2}-4 M r+a^{2}}{r^{2}+a^{2}}-\frac{3 r^{2} \Delta}{\left(r^{2}+a^{2}\right)^{2}}\right) .
\end{aligned}
$$

This potential depends nontrivially on the energy $\omega^{2}$ of the field. Now, from Eq. (6.7) it is obvious that in the proximity of the horizon, defined by $\Delta\left(r_{\mathcal{H}}\right)=0$, the potential is negligible with respect to the "energy" of the field $\omega^{2}$. Accordingly, we can solve Eq. (6.7) to first order in $|V| / \omega^{2}$, and write the solution

$$
H_{\ell \omega}=\exp \left( \pm \omega r^{*}\right)+(\text { higher order terms }) .
$$

We choose appropriate boundary conditions for the solution of Eq. (6.7), analogously to Matzner's choice [17 for the Schwarzschild case $(a=0)$. Thus, the minus sign in the $\omega$-mode expansion of $\Phi$ and in Eq. (6.8) is singled out. This is a result of the demand that only ingoing waves exist at the one-way membrane $r^{*} \rightarrow-\infty$.

Before undertaking the task of verifying that the perturbation does leave the horizon area unchanged, we have to verify that the solution is indeed physical, namely invariants of the curvature are bounded on the horizon. To do so we look, as before, at terms such as $\nabla_{\alpha} \Phi \nabla^{\alpha} \Phi$. A direct calculation of the last term delivers the following result: 


$$
\nabla_{\alpha} \Phi \nabla^{\alpha} \Phi=\frac{1}{\rho^{2}\left(r^{2}+a^{2}\right)}\left(a^{2} \omega^{2} \sin \psi^{2} \sin \theta^{2} S_{\ell 0}{ }^{2}+\omega r \sin 2 \psi S_{\ell 0}+\cos \psi^{2}\left(\frac{\partial S_{\ell 0}}{\partial \theta}\right)^{2}\right)+\cdots
$$

where $\psi=\omega\left(r^{*}+t\right)$. Surely, the last result indicates that the solution we have found does not cause any singularities. Thus, the solution can be taken to be physical.

Finally, following the chain of reasoning that led us to the derivation of Eq. (6.4), we find that

$$
T_{\alpha \beta} l^{\alpha} l^{\beta}=\left(l^{t} \partial \Phi / \partial t+l^{r} \partial \Phi / \partial r\right)^{2} \propto \frac{\omega^{2} S_{\ell}^{2} \sin (\psi)^{2}}{r^{2}+a^{2}}+\cdots
$$

This finding is in accordance with the results obtained in the previous sections. Thus we find yet another example of the adiabatic invariance of the black hole horizon area.

\section{BLACK HOLE DISTURBED BY ELECTRIC CHARGES}

Here we shall examine the influence of an electromagnetic radiation on the black hole horizon area. In order to sidestep the problems of electromagnetic interactions between the black hole and the charges which produce the electromagnetic waves, we assume that the black hole is neutral. In the first subsection we formulate the problem. We make use of the possibility to describe the Schwarzschild space-time as a conformally flat spacetime, occupied by a "medium" which has a non-trivial permeabilities. In the following two last subsections we discuss the effect of the electromagnetic radiation on the horizon area in the static case and in the dynamic case.

\section{A. Formulation of the problem}

Following the footsteps of [19], Maxwell's equations can be cast into a simple form if we write the Schwarzschild metric in isotropic coordinates. Consider the coordinate transformation $\rho$ defined by $r \equiv M+\rho+M^{2} / 4 \rho$ and let $f(\rho) \equiv 1-2 M / r(\rho)$ and $u(\rho) \equiv r(\rho) / \rho$, then the metric reads

$$
d s^{2}=-f(\rho) d t^{2}+u(\rho)^{2}\left(d \rho^{2}+\rho^{2} d \theta^{2}+\rho^{2} \sin \theta^{2} d \varphi^{2}\right) .
$$

As far as the electromagnetic phenomena are concerned we can think of the space-time as Minkowskian, occupied by a "medium" which is characterized by dielectric and magnetic permeability tensors $\epsilon_{i k}=\mu_{i k} \equiv n(\rho) \delta_{i k}$ where

$$
n(\rho) \equiv u(\rho) / \sqrt{f(\rho)} .
$$

$n(\rho)$ can be viewed as an index of refraction induced by gravity. Note that $n(\rho)$ is everywhere positive definite, and diverges as $r \rightarrow 2 M$. Maxwell's equations can then be written as

$$
\begin{aligned}
& \frac{1}{\imath} \vec{\nabla} \times \vec{F}^{ \pm}= \pm n \frac{\partial \vec{F}^{ \pm}}{\partial t}, \\
& \vec{\nabla} \cdot\left(n \vec{F}^{ \pm}\right)=0,
\end{aligned}
$$

where $\vec{F}^{ \pm}=\vec{E} \pm \imath \vec{H} . \vec{E}$ and $\vec{H}$ are the electric and magnetic fields in the medium respectively. The constitutive equations can be written as

$$
\begin{aligned}
& \vec{D}=n(\rho) \vec{E}, \\
& \vec{B}=n(\rho) \vec{H} .
\end{aligned}
$$

Note that due to the static nature of the spacetime these constitutive equations do not include "gyroscopic" terms such as $\vec{G} \times \vec{E}$ or $\vec{G} \times \vec{H}$, where $\vec{G}$ represents the vorticity of the timelike killing vector. $\vec{\nabla}$ is taken in the last equation as the usual gradient operator in 3-dimensions, $\hat{\rho} \partial / \partial \rho+\hat{\theta}(1 / \rho) \partial / \partial \theta+\hat{\varphi}(1 / \rho \sin \theta) \partial / \partial \varphi$. $\hat{\rho}, \hat{\theta}$ and $\hat{\varphi}$ are unit vectors in the radial, polar and azimuthal directions respectively.

Consider now the representation

$$
\vec{F}^{ \pm}(\vec{\rho}, t)=\sum_{k} \int d \omega e^{-\imath \omega t} \sum_{\sigma=0, e, \mu} F_{k}^{ \pm(\sigma)}(\rho, \omega) \vec{Y}_{k}^{(\sigma)}(\hat{\rho}),
$$


$k$ is an alias for $(\ell, m) . \ell$ and $m$ have the usual range. $\vec{Y}_{k}^{(e)}$ and $\vec{Y}_{k}^{(\mu)}$ are the transverse and $\vec{Y}_{k}^{(0)}$ the longitudinal vector spherical harmonics [20]. They may be expressed by

$$
\begin{aligned}
\vec{Y}_{k}^{(0)} & =\hat{\rho} Y_{k}(\hat{\rho}), \\
\vec{Y}_{k}^{(e)} & =\vec{\nabla}_{\hat{\rho}} Y_{k}(\hat{\rho}), \\
\vec{Y}_{k}^{(\mu)} & =\hat{\rho} \times \vec{\nabla}_{\hat{\rho}} Y_{k}(\hat{\rho}) .
\end{aligned}
$$

When one substitutes this in Maxwell's equations, Eq. (7.3), one gets

$$
\begin{aligned}
-\sqrt{\ell(\ell+1)} F_{k}^{ \pm(\mu)} & = \pm \omega n(\rho) F_{k}^{ \pm(0)}, \\
-\frac{d}{d \rho}\left(\rho F_{k}^{ \pm(\mu)}\right) & = \pm \omega \rho n(\rho) F_{k}^{ \pm(e)}, \\
\frac{d}{d \rho}\left(\rho F_{k}^{ \pm(e)}\right)-\sqrt{\ell(\ell+1)} F_{k}^{ \pm(0)} & = \pm \omega \rho n(\rho) F_{k}^{ \pm(\mu)}, \\
\frac{d F_{k}^{ \pm(0)}}{d \rho}+\frac{d}{d \rho}\left(\ln \left(\rho^{2} n\right)\right) F_{k}^{ \pm(0)}+\ell(\ell+1) F_{k}^{ \pm(e)} & =0 .
\end{aligned}
$$

An indication of the relevance of the solution to the physical problem at hand can be obtained by examining the invariants that may be constructed from the energy-momentum tensor of the perturbation

$$
T_{\mu}^{\nu}=\frac{1}{4 \pi \sqrt{-g}}\left(F_{\mu \alpha} \mathcal{H}^{\beta \alpha} \delta_{\beta}^{\nu}-\frac{1}{4} \delta_{\mu}^{\nu} F_{\alpha \beta} \mathcal{H}^{\alpha \beta}\right),
$$

where we define the usual field strength, $F_{\alpha \beta} \rightarrow(\vec{E}, \vec{B})$ and the pseudo-tensor $\mathcal{H}^{\alpha \beta} \equiv \sqrt{-g} F^{\alpha \beta} \rightarrow(-\vec{D}, \vec{H})$. Note that $\sqrt{-g}=u^{4} / n$. Every invariant of this kind must be bounded for a physically acceptable solution. The analysis can be simplified much using the following arguments. $T_{\mu}^{\nu}$ may be seen as a $4 \times 4$ real matrix [21]. Thus, any invariant constructed from $T_{\mu}^{\nu}$ is actually the trace of some power of $\mathbf{T}$. For example, $T_{\alpha}^{\alpha}=\operatorname{Tr} \mathbf{T} \equiv 0, T_{\beta}^{\alpha} T_{\alpha}^{\beta}=\operatorname{Tr} \mathbf{T}^{2}$, $T_{\beta}^{\alpha} T_{\gamma}^{\beta} T_{\alpha}^{\gamma}=\operatorname{Tr} \mathbf{T}^{3}$ etc. Now, in this matrix notation $T_{\mu}^{\nu}$ can be written as

$$
\mathbf{T}=\frac{1}{4 \pi \sqrt{-g}}\left(\mathbf{M}-\frac{1}{4} \operatorname{Tr} \mathbf{M} 1\right)
$$

where $M_{\mu}^{\nu} \equiv F_{\mu}^{\alpha} \mathcal{H}_{\alpha}^{\nu}$ or in matrix notation $\mathbf{M} \equiv \mathbf{F} \cdot \mathcal{H}$ and 1 is a $4 \times 4$ identity matrix. The trace of any power of $\mathbf{T}$ is simply the trace of some polynomial function of $\mathbf{M}$, where the polynomials coefficients may be powers of $\operatorname{Tr} \mathbf{M}$. Now, since the trace operator is linear, it is sufficient to look at expressions like $\operatorname{Tr} \mathbf{M}, \operatorname{Tr} \mathbf{M}^{2}, \operatorname{Tr} \mathbf{M}^{3}$ etc. These in turn are simply the sum of the eigenvalues of $\mathbf{M}$ raised to the appropriate power. Hence, a prerequisite for the boundness of the invariants of the curvature is that the eigenvalues of $\mathbf{M}$ vanishe at least as fast as $1 / n$ as we approach the horizon. It turns out that $\mathbf{M}$ has only two distinct eigenvalues which have each a two-fold degeneracy. These are

$$
\lambda_{1}=\frac{1}{4}\left(\left\|\vec{F}^{+}\right\|+\left\|\vec{F}^{-}\right\|\right)^{2}, \quad \lambda_{2}=\frac{1}{4}\left(\left\|\vec{F}^{+}\right\|-\left\|\vec{F}^{-}\right\|\right)^{2}
$$

where $\left\|\vec{F}^{ \pm}\right\| \equiv\left(\vec{F}^{ \pm} \cdot \vec{F}^{ \pm}\right)^{1 / 2}$. Now if one uses the definition of the vector spherical harmonic Eq. (7.6) it is plain to see that $\vec{Y}_{k_{1}}^{(e)} \cdot \vec{Y}_{k_{2}}^{(e)}=\vec{Y}_{k 1}^{(\mu)} \cdot \vec{Y}_{k_{2}}^{(\mu)}$ for every $k$, and since the vector spherical harmonics form an orthogonal base, $\left\|\vec{F}^{ \pm}\right\|$can be reduced to

$$
\begin{aligned}
\left\|\vec{F}^{ \pm}\right\|^{2}= & \Re \sum_{k_{1}, k_{2}} \int d \omega_{1} d \omega_{2} e^{-\imath\left(\omega_{1}+\omega_{2}\right) t} \\
& \left(Y_{k_{1}} Y_{k_{2}} F_{k_{1}}^{ \pm(0)} F_{k_{2}}^{ \pm(0)}+\vec{\nabla} Y_{k_{1}} \cdot \vec{\nabla} Y_{k_{2}} \sum_{\sigma=e, \mu} F_{k_{1}}^{ \pm(\sigma)} F_{k_{2}}^{ \pm(\sigma)}\right) .
\end{aligned}
$$

In the following sections we will use this expression to verify that our solution is physically meaningful. 
After the solution is shown to be physical, we will test whether the horizon area is left unchanged under the disturbance. This will be confirmed if the term $4 \pi T_{\alpha \beta} l^{\alpha} l^{\beta}$ vanishes on the horizon. In Sec. III $l^{\alpha}$, the null generator of the horizon was shown to be proportional to the vector $N^{\alpha}$. Now, under the coordinates transformation to the isotropic coordinates system, the vector $N^{\alpha}$ transforms to

$$
N^{\alpha} \equiv(1,1-2 M / r, 0,0) \rightarrow(1,1 / n, 0,0)
$$

Thus, a straightforward calculation leads to the following result:

$$
4 \pi T_{\alpha \beta} l^{\alpha} l^{\beta} \propto \frac{1}{u^{2}} \Re\left(F_{\theta}{ }^{+}+\imath F_{\phi}^{+}\right)\left(F_{\theta}{ }^{-}-\imath F_{\phi}{ }^{-}\right)
$$

and after substitution of $\vec{F}^{ \pm}$from Eq. 7.5 , one obtains

$$
F_{\theta}^{ \pm} \pm \imath F_{\phi}^{ \pm}=\sum_{k} \int d \omega \frac{e^{-\imath \omega t}}{\rho}\left(F_{k}^{ \pm(e)} \pm \imath F_{k}^{ \pm(\mu)}\right) \mathcal{D}^{ \pm} Y_{k}
$$

where $\mathcal{D}^{ \pm}$is the differential operator $\partial / \partial \theta \pm(\iota / \sin \theta) \partial / \partial \varphi$. All the above is general. In the following subsection we shall discuss the static problem. Finally, in the last subsection we shall retain the time dependence of the fields and solve the dynamical problem.

\section{B. The Static Problem}

When the electromagnetic fields produced by the charges are static, Maxwell's equations [Eq. (7.7)] can be reduced to

$$
\begin{aligned}
\sqrt{\ell(\ell+1)} F_{k}^{ \pm(\mu)} & =0, \\
\frac{d}{d \rho}\left(\rho F_{k}^{ \pm(\mu)}\right) & =0, \\
\frac{d}{d \rho}\left(\rho F_{k}^{ \pm(e)}\right)-\sqrt{\ell(\ell+1)} F_{k}^{ \pm(0)} & =0, \\
\frac{d F_{\ell m}^{ \pm(0)}}{d \rho}+\frac{d}{d \rho}\left(\ln \left(\rho^{2} n\right)\right) F_{k}^{ \pm(0)}+\ell(\ell+1) F_{k}^{ \pm(e)} & =0 .
\end{aligned}
$$

The first two equations simply state that $F_{k}^{ \pm(\mu)}=0$ for every $k$. The last two equations can be combined to give

$$
\begin{gathered}
\frac{d^{2}}{d \rho^{2}}\left(\rho F_{k}^{ \pm(e)}\right)+\frac{d}{d \rho}\left(\ln \left(\rho^{2} n\right)\right) \frac{d}{d \rho}\left(\rho F_{k}^{ \pm(e)}\right)+(\ell(\ell+1))^{3 / 2} F_{k}^{ \pm(e)}=0 \\
F_{k}^{ \pm(0)}=\frac{1}{\sqrt{\ell(\ell+1)}} \frac{d}{d \rho}\left(\rho F_{k}^{ \pm(e)}\right) .
\end{gathered}
$$

$F_{k}^{ \pm(0)}$ is given by the second equation of Eq. (7.16) after the first equation is solved for $F_{k}^{ \pm(e)}$.

After the substitution of $n(\rho)$ and the introduction of a new function $X_{k}^{ \pm(e)}=\rho F_{k}^{ \pm(e)}$, we can write the equation for $F_{k}^{ \pm(e)}$ in the following form

$$
\frac{d^{2} X_{k}^{ \pm(e)}}{d \rho^{2}}+2 \frac{\rho-M}{\left(\rho+\frac{M}{2}\right)\left(\rho-\frac{M}{2}\right)} \frac{d X_{k}^{ \pm(e)}}{d \rho}+(\ell(\ell+1))^{3 / 2} \frac{X_{k}^{ \pm(e)}}{\rho}=0 .
$$

Subsequently we will be interested in the behavior of the electromagnetic fields in the proximity of the horizon. Therefore, we look for an approximate solution to Eq. (7.17) near $\rho=M / 2$. We introduce a new variable $x=\rho-M / 2$ and a new function $X_{k}^{ \pm(e)}(x)=x z_{k}^{ \pm(e)}(x)$. Then Eq. (7.17) assumes the following form

$$
x^{2} \frac{d^{2} z_{k}^{ \pm(e)}}{d x^{2}}+x \frac{d z_{k}^{ \pm(e)}}{d x}+\left(\alpha x^{2}-1\right) z_{k}^{ \pm(e)} \simeq 0
$$


with $\alpha=2(\ell(\ell+1))^{3 / 2} / M$. This equation is the familiar Bessel equation of order 1 with the two independent solutions $J_{1}(\sqrt{\alpha} x)$ and $Y_{1}(\sqrt{\alpha} x)$. Convergence of the curvature invariant, Eq $(7.11)$, forbids us from considering the $Y_{1}(\sqrt{\alpha} x)$ solution as a physical one since it diverges at $x=0$, namely at the horizon. As in the former cases, this divergence is unacceptable since it will induce divergences in the invariants of the curvature, thus disqualifying the perturbation method.

Using Eq. (7.16), it is now possible to show that

$$
\begin{aligned}
F_{k}^{ \pm(\mu)} & \equiv 0 \\
F_{k}^{ \pm(e)} & \simeq \frac{x}{\rho} C_{k}^{ \pm} J_{1}(\sqrt{\alpha} x) \\
F_{k}^{ \pm(0)} & \simeq \frac{C_{k}^{ \pm}}{\sqrt{\ell(\ell+1)}}\left(J_{1}(\sqrt{\alpha} x)-\frac{\sqrt{\alpha} x}{2}\left(J_{0}(\sqrt{\alpha} x)-J_{2}(\sqrt{\alpha} x)\right)\right) .
\end{aligned}
$$

Hence, according to Eq. (7.13) and Eq. (7.14) (with $\omega=0$ ) the behavior of $4 \pi T_{\alpha \beta} l^{\alpha} l^{\beta}$ is governed solely by the behavior of $F_{k}^{ \pm(e)}$ near the horizon. Now, for $x \rightarrow 0, J_{1}(\sqrt{\alpha} x)$ is of $O(x)$. Therefore, $F_{k}^{ \pm(e)}$ is of $O\left(x^{2}\right)$ and $F_{k}^{ \pm(0)}$ is of $O(x)$. This implies that $4 \pi T_{\alpha \beta} l^{\alpha} l^{\beta}$ is of order $O\left(x^{4}\right)$. Thus the horizon area does not change under the action of the static electromagnetic fields.

Since the adiabatic invariance of an object is primarily meaningful in the context of dynamic processes, we turn now to deal with the dynamic problem. Basically, we will consider the process in which the charges are brought adiabatically from infinity through the potential barrier of the black hole into the immediate surroundings of the horizon. This will be dealt with in the next subsection.

\section{Moving the Charges Around}

First we introduce a new coordinate $r^{*}$ defined by $d r^{*} / d \rho \equiv n(\rho)$. Then $r^{*}(\rho)$ can be identified with Wheeler's "tortoise" coordinate [14]; $r^{*}=r+2 M \ln (r / 2 M-1)$. Moreover, as usual, we define new radial functions $X_{k}^{ \pm(\sigma)}\left(r^{*}, \omega\right)=\rho F_{k}^{ \pm(\sigma)}$. In terms of these new radial functions the radial parts of Maxwell's equations reads

$$
\begin{aligned}
-\frac{d^{2} X_{k}^{ \pm(\mu)}}{d r^{* 2}} & +\left(1-\frac{2 M}{r}\right) \frac{\ell(\ell+1)}{r^{2}} X_{k}^{ \pm(\mu)}=\omega^{2} X_{k}^{ \pm(\mu)}, \\
X_{k}^{ \pm(e)} & =\mp(1 / \omega) \frac{d X_{k}^{ \pm(\mu)}}{d r^{*}}, \\
X_{k}^{ \pm(0)} & =\mp(1 / \omega) \sqrt{\ell(\ell+1)} \frac{X_{k}^{ \pm(\mu)}}{\rho n} .
\end{aligned}
$$

We are interested in a solution in the region where $\omega^{2}$ is much larger than the potential barrier, namely in the region where

$$
\omega^{2} \gg\left(1-\frac{2 M}{r}\right) \frac{\ell(\ell+1)}{r^{2}}
$$

An approximate solution is

$$
\begin{aligned}
& X_{k}^{ \pm(\mu)} \simeq C_{k}^{ \pm}(\omega) e^{-\imath \omega r^{*}} \\
& X_{k}^{ \pm(e)} \simeq \pm \imath C_{k}^{ \pm}(\omega) e^{-\imath \omega r^{*}} \\
& X_{k}^{ \pm(0)} \simeq \mp \sqrt{\ell(\ell+1)} \frac{C_{k}^{ \pm}(\omega) e^{-\imath \omega r^{*}}}{\omega \rho n} .
\end{aligned}
$$

A useful by-product of the fact that $1 / n$ vanishes on the horizon, is that the longitudinal component of the electromagnetic fields vanishes as $\sqrt{1-2 M / r}$ when $r \rightarrow 2 M$.

After substitution of $X^{ \pm(\sigma)}$ from Eq. (7.22) in Eq. (7.11), it is evident that the summation over $\sigma$ vanishes identically, regardless of the magnitude of the frequency dependent coefficients, $C_{k}^{ \pm}(\omega)$. And as mentioned, the longitudinal term

$F_{k_{1}}^{ \pm(0)} F_{k_{2}}^{ \pm(0)}$ is proportional to $1 / n^{2}$. Hence, the multiplication of this term by $n$ does not cause any trouble. In fact as 
we approach the horizon the contribution from this term to the perturbation of the metric becomes negligible. This shows that every invariant of the type we considered vanishes on the horizon in spite of the divergence of $n$ there. Of course, globally these invariants may still have an effect on the geometry. In particular the geometry of the horizon can get disturbed.

Now, since the perturbation is supposed to be weak, we are limited to small frequencies, namely long wavelengths compared to the size of the hole, $\lambda>>2 M$. Additionally the coefficients $C_{k}^{ \pm}(\omega)$ must be bounded everywhere and are assumed to be smooth functions of $\omega$. As before, consider a solution composed of a single mode. Then, substitution of this solution, Eq. (7.22), in Eq. (7.11) gives

$$
\left\|\vec{F}^{ \pm}\right\|^{2} \simeq \ell(\ell+1) \Re\left(\frac{C_{k}^{ \pm}(\omega)}{\omega}\right)^{2}\left(P_{\ell} \frac{\cos \psi}{\rho^{2} n}\right)^{2}=O\left(\left[\frac{C_{k}(\omega)}{\omega}\right]^{2}\right)
$$

where $\psi=\omega\left(r^{*}+t\right)-m \varphi$. It is evident from above that at a small radial distance from the horizon the electromagnetic fields induce an oscillatory disturbance of $O\left(\left[C_{k}(\omega) / \omega\right]^{2}\right)$. The disturbance vanishes on the horizon. The limit $\omega \rightarrow 0$ was dealt with in the previous subsection and was found to exist. Now, how does the horizon area change when the weak perturbation is turned on? Consider again a solution composed of a single mode. Then, the substitution of this solution in Eq. (7.14) and Eq. (7.13) gives

$$
4 \pi T_{\alpha \beta} l^{\alpha} l^{\beta} \propto\left(\frac{2 \cos \psi}{\rho^{2} u}\right)^{2}\left(\left(\frac{d P_{\ell}}{d \theta}\right)^{2}-\left(\frac{m P_{\ell}}{\sin \theta}\right)^{2}\right) \Re\left(C_{k}^{+}(\omega) C_{k}^{-}(\omega)\right)=O\left(C_{k}(\omega)^{2}\right) .
$$

Obviously, this result states that the perturbation to the horizon area is a quantity of higher order in $\omega$ than the perturbation to the geometry as quantified by the invariants calculated previously. Thus horizon area is again seen to be an adiabatic invariant.

ACKNOWLEDGMENTS The author would like to thank Professor J. D. Bekenstein for proposing this problem and for his suggestions and advice, and to S. Hod for helpful discussions. This work is supported by a grant from the Israel Science Foundation established by the Israel Academy of Sciences.

[1] S. W. Hawking, Phys. Rev. Lett. 26, 1344 (1971).

[2] J. D. Bekenstein, Phys. Rev. D 7, 2333 (1973); S. W. Hawking, Commun. Math. Phys. 43, 212 (1975).

[3] V. I. Arnold, Mathematical Methods of Classical Mechanics 2nd ed. (Springer-Verlag, New York, 1989).

[4] L. D. Landau and E. M. Lifshits, Mechanics: Course of Theoretical Physics, 3rd ed. (Pergamon, Oxford, New York 1976).

[5] For example see M. Born, Atomic Physics 8th ed. (Blackie, London, 1969).

[6] K. S. Thorne, R. H. Price and D. A. MacDonald, Black Holes: The Membrane Paradigm (Yale University Press, London, 1986).

[7] M. K. Parikh and F. Wilczek, Phys. Rev. D. (to be published), gr-qc/9712077.

[8] C. Teitelboim, Phys. Rev. D. 53, 2870 (1996).

[9] J. D. Bekenstein, Lett. Nuovo Cimento 11, 467 (1974); V. F. Mukhanov, JETP Lett. 44, 63 (1986); V. F. Mukhanov in Complexity, Entropy and the Physics of Information: SFI Studies in the Sciences of Complexity, edited by W. H. Zurek (Addison-Wesley, New York, 1990), Vol. III, p. 47 ; J. D. Bekenstein and V. F. Mukhanov, Phys. Lett. B 360, 7 (1995); J. D. Bekenstein, in Proceedings of the XVII Brazilian National Meeting on Particles and Fields, edited by A. J. da Silva et. al. (Brazilian Physical Society 1996); J. D. Bekenstein, in Proceedings of the VIII Marcel Grossmann Meeting on General Relativity, edited by T. Piran and R. Ruffini, (World Scientific, Singapore 1998).

[10] J. D. Bekenstein, in Black Holes, Gravitational Radiation and the Universe, edited by B. Bhawal and B. Iyer (Kluwer, Dordrecht 1998).

[11] S. W. Hawking and J. B. Hartle, Commun. Math. Phys. 27, 283 (1972).

[12] E. Newman and R. Penrose, J. Math. Phys. 3, 566 (1962).

[13] F. A. E. Pirani, in Lectures on General Relativity: Brandeis Summer Institute in Theoretical Physics, edited by S. Deser and K. W. Ford (Prentice-Hall, Englewood Cliffs, NJ, 1965), Vol. I p. 249.

[14] C. W. Misner, K. S. Thorne and J. A. Wheeler, Gravitation (Freeman, San Francisco, 1973).

[15] J. Mathews and R. L. Walker, Mathematical Methods of Physics, 2nd ed. (Benjamin, Menlo Park, California, 1970).

[16] J. Bičák, Gen. Relativ. Gravit. 3, 331 (1972).

[17] R. A. Matzner, Phys. Rev. D 9, 163 (1968). 
[18] E. D. Fackerell et al. Phys. Rev. D 5, 1913 (1971).

[19] A. M. Volkov, A. A. Izmest'ev and G. V. Skrotskii, Sov. Phys. JETP 32, 686 (1971); B. Mashhoon, Phys. Rev. D 7, 2807 (1973).

[20] J. D. Jackson, Classical Electrodynamics 2nd ed. (Wiley, New York, 1975).

[21] Further implications of this approach may be found in A. E. Mayo and J. D. Bekenstein, Phys. Rev. D 54, 5059 (1996). 